University of Nebraska - Lincoln

DigitalCommons@University of Nebraska - Lincoln

Faculty Publications from the Department of Electrical \& Computer Engineering, Department Electrical and Computer Engineering

$7-1-2003$

\title{
Progress in spectroscopic ellipsometry: Applications from vacuum ultraviolet to infrared
}

James N. Hilfiker

J. A. Woollam Co.

Corey L. Bungay

J. A. Woollam Co.

Ron A. Synowicki

J. A. Woollam Co.

Thomas E. Tiwald

J. A. Woollam Co.

Craig M. Herzinger

J. A. Woollam Co.

See next page for additional authors

Follow this and additional works at: https://digitalcommons.unl.edu/electricalengineeringfacpub

Part of the Electrical and Computer Engineering Commons

Hilfiker, James N.; Bungay, Corey L.; Synowicki, Ron A.; Tiwald, Thomas E.; Herzinger, Craig M.; Johs, Blaine; Pribil, Greg K.; and Woollam, John A., "Progress in spectroscopic ellipsometry: Applications from vacuum ultraviolet to infrared" (2003). Faculty Publications from the Department of Electrical and Computer Engineering. 10.

https://digitalcommons.unl.edu/electricalengineeringfacpub/10

This Article is brought to you for free and open access by the Electrical \& Computer Engineering, Department of at DigitalCommons@University of Nebraska - Lincoln. It has been accepted for inclusion in Faculty Publications from the Department of Electrical and Computer Engineering by an authorized administrator of DigitalCommons@University of Nebraska - Lincoln. 


\section{Authors}

James N. Hilfiker, Corey L. Bungay, Ron A. Synowicki, Thomas E. Tiwald, Craig M. Herzinger, Blaine Johs, Greg K. Pribil, and John A. Woollam 


\title{
Progress in spectroscopic ellipsometry: Applications from vacuum ultraviolet to infrared
}

\author{
James N. Hilfiker, ${ }^{\text {a) }}$ Corey L Bungay, Ron A. Synowicki, Thomas E. Tiwald, \\ Craig M. Herzinger, Blaine Johs, Greg K. Pribil, and John A. Woollam \\ J. A. Woollam Co., Inc., 645 Main Street, Suite 102, Lincoln, Nebraska 68508
}

(Received 30 December 2002; accepted 3 March 2003; published 30 June 2003)

\begin{abstract}
Spectroscopic ellipsometry (SE) is a noncontact and nondestructive optical technique for thin film characterization. In the past $10 \mathrm{yr}$, it has migrated from the research laboratory into the semiconductor, data storage, display, communication, and optical coating industries. The wide acceptance of $\mathrm{SE}$ is a result of its flexibility to measure most material types: dielectrics, semiconductors, metals, superconductors, polymers, biological coatings, and even multilayers of these materials. Measurement of anisotropic materials has also made huge strides in recent years. Traditional SE measurements cover the ultraviolet, visible, and near infrared wavelengths. This spectral range is now acquired within seconds with high accuracy due to innovative optical configurations and charge coupled device detection. In addition, commercial SE has expanded into both the vacuum ultraviolet (VUV) and midinfrared (IR). This wide spectral coverage was achieved by utilizing new optical elements and detection systems, along with UV or Fourier transform IR light sources. Modern instrumentation is now available with unprecedented flexibility promoting a new range of possible applications. For example, the VUV spectral region is capable of characterizing lithographic materials for $157 \mathrm{~nm}$ photolithography. The VUV also provides increased sensitivity for thin layers (e.g., gate oxides or self-assembled monolayers) and allows investigation of high-energy electronic transitions. The infrared spectral region contains information about semiconductor doping concentration, phonon absorption, and molecular bond vibrational absorptions. In this work, we review the latest progress in SE wavelength coverage. Areas of significant application in both research and industrial fields will be surveyed, with emphasis on wavelength-specific information content. (C) 2003 American Vacuum Society.
\end{abstract}

[DOI: $10.1116 / 1.1569928]$

\section{INTRODUCTION}

Ellipsometry is an optical measurement of the polarization change occurring when light interacts (reflection/ transmission) with materials. It is commonly applied to bulk substrates and single or multilayer coatings. The measurement is described by two parameters, $\Psi$ and $\Delta$, that represent an amplitude and phase change, respectively. Equation (1) relates these parameters to the complex Fresnel coefficients for $p$ - and $s$-polarized light:

$$
\tan (\Psi) e^{i \Delta}=\frac{\widetilde{R}_{p}}{\widetilde{R}_{s}} .
$$

Reflection from a bulk, isotropic substrate with no coatings or overlayers presents a simple case where the measured ellipsometry parameters can be inverted to determine the real and imaginary dielectric function ( $\varepsilon_{1}$ and $\varepsilon_{2}$, respectively). However, this case is uncommon due to surface oxidation or roughness on most substrates. More often, the material properties are determined from a model-based regression. A full description of this process can be found elsewhere. ${ }^{1-6} \mathrm{~A}$ model-based regression is limited to the information content

${ }^{a)}$ Electronic mail: jhilfiker@jawoollam.com available from the experimental data. For this reason, ellipsometry is often performed at multiple angles and wavelengths to increase the available information.

Angle of incidence variations will change the path length of the probe light through a thin film. Even when an additional angle does not contain unique new information, it can always serve to confirm model validity. The correct thickness and optical constants must describe the interaction between light and the film for all angles of incidence. Multiple angles often contain new information about the material properties of semiabsorbing films, multilayer structures (especially if one layer is semiabsorbing), and anisotropic materials. For semiabsorbing films, a different path length changes the amount of absorbed light. For anisotropic materials, an angle variation will change the electric-field interaction along different optical axes.

Spectroscopic measurements provide additional information content for each new wavelength. While the film thickness remains constant irregardless of wavelength, the optical constants will change across the spectrum. The optical constant dispersion carries information about many material properties. Spectroscopic ellipsometry (SE) has a wide variety of applications. Significant instrument development in the past decade has focused on increasing wavelength range and improving wavelength resolution. To accomplish this, different light sources and detectors have been utilized as 


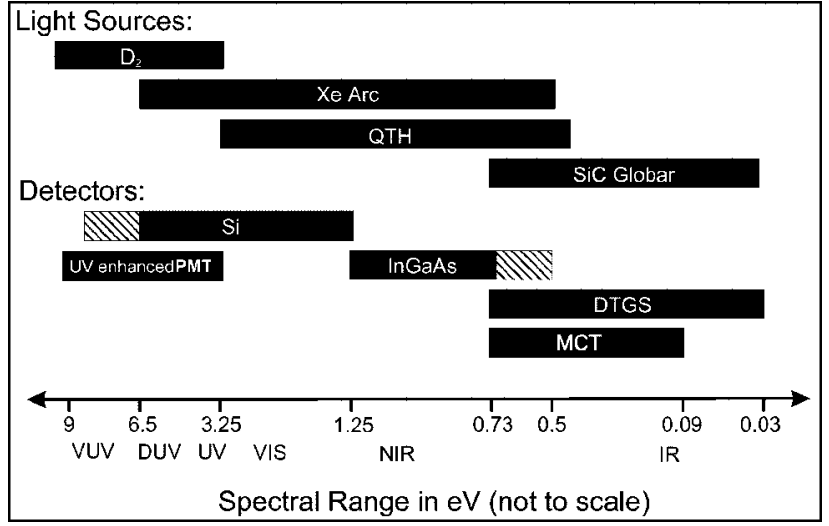

FIG. 1. Wavelength ranges of common light sources and detectors used in spectroscopic ellipsometers.

shown in Fig. 1. Often, these elements are combined to increase the wavelength range of a single SE system. In addition, two relatively new wavelength regions have been incorporated into SE systems-the vacuum ultraviolet (VUV) and infrared (IR). This discussion will focus on the importance of wavelength range for many applications. Particular importance will be placed on the role of VUV and IR ellipsometry for new potential applications.

\section{OVERVIEW OF TYPICAL SE APPLICATIONS}

SE characterization has been applied to a wide range of industrial and research applications. It can measure any material type on most substrates; provided a specular reflection is produced. SE also gained popularity for ultrathin film measurements due to high sensitivity from the "phase" component, $\Delta$. This has become increasingly important for gate dielectrics in the semiconductor industry, diamond-like carbon in magnetic recording devices, and monolayer biological films.

While SE is used for all types of materials, light must penetrate a film to provide thickness information. Figure 2 illustrates an optical measurement of a coated substrate. On the left, light penetrates the film and returns to interfere with light reflecting from the surface. This interference allows SE to determine film thickness. Thickness information is not available if the measurement beam is absorbed before returning to the surface (second case in Fig. 2).

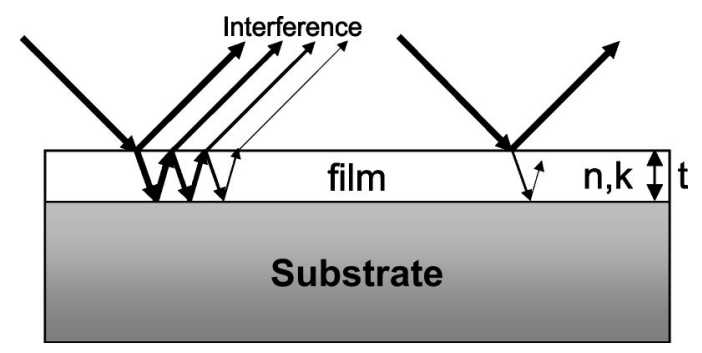

FIG. 2. Ellipsometry measurements of a thin film rely on interference (left) to determine thickness $t$. If the film is absorbing (right) at all measured wavelengths, the thickness is not determined. Both cases contain information about the film optical constants $(n, k)$.

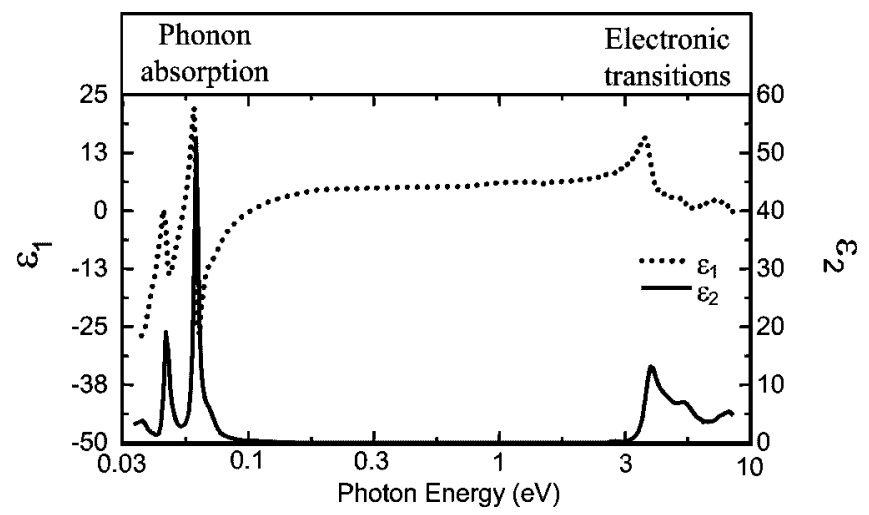

FIG. 3. Dielectric function measured for $\mathrm{TiO}_{2}$ from IR to VUV wavelengths. This material is absorbing at both IR and UV due to phonon absorptions and electronic transitions, respectively (see Ref. 7).

To determine film thickness, it is important to measure a transparent wavelength region for the film. Dielectrics, polymers and even semiconductors are generally transparent at near IR (NIR) wavelengths. These materials often absorb light in the UV and sometimes the visible due to electronic transitions. Many will also absorb in the IR due to phonon, molecular vibration, or free-carrier absorption. Figure 3 shows the measured dielectric function for $\mathrm{TiO}_{2} \cdot{ }^{7}$ In the UV, this material is absorbing due to valence electron transitions. The material is transparent across the visible and NIR until phonon absorption occurs in the IR. Thus, the thickness of a dielectric $\mathrm{TiO}_{2}$ film can be measured with SE across the visible and near infrared spectral region-where it remains transparent.

There are many material properties that are best measured with a specific wavelength region. The composition of a crystalline semiconductor produces a distinct position and shape for the electronic transitions. Measurements in the absorbing region can be used to determine the optical response for a semiconductor. This relates to the composition, as shown in Fig. 4 for $\mathrm{Al}_{x} \mathrm{Ga}_{1-x}$ As. The direct band gap and higher electronic transitions shift toward blue with increasing aluminum concentration. This effect has been used to study binary semiconductors, such as $\mathrm{Si}_{x} \mathrm{Ge}_{1-x},{ }^{8}$ as well as ternary and quaternary semiconductors. The composition of

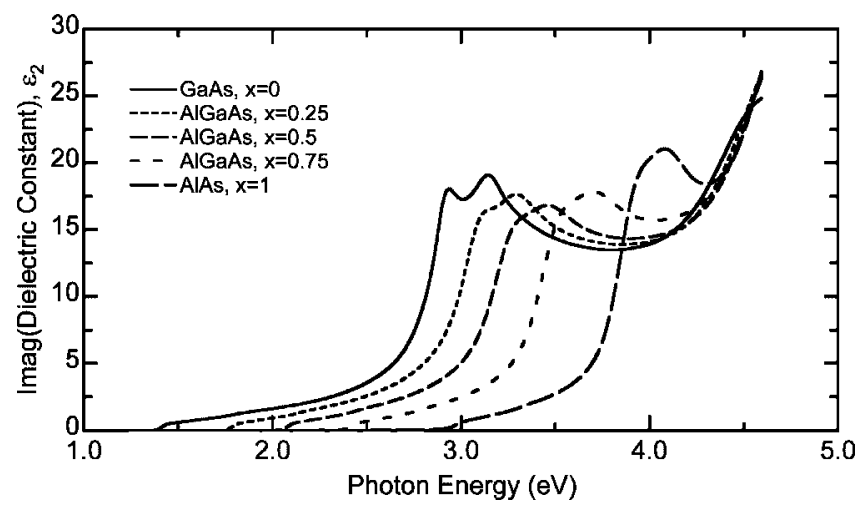

FIG. 4. Critical points of $\mathrm{Al}_{x} \mathrm{Ga}_{1-x}$ As shift toward blue with increasing $\mathrm{Al}$ concentration $x$. 


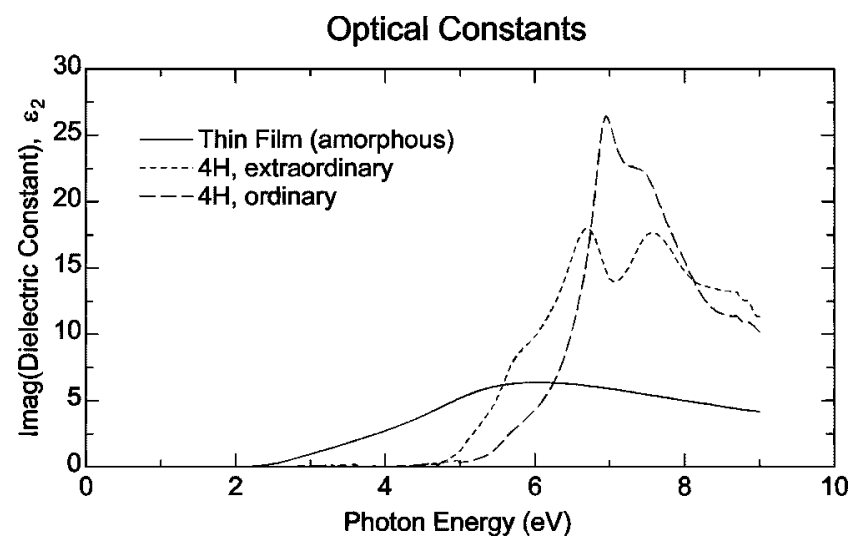

FIG. 5. Measurement of $\mathrm{SiC}$ optical properties depend on crystallinity. Dashed curves represent ordinary and extraordinary axis for $4 \mathrm{H} \mathrm{SiC}$, while the solid curve was measured from an amorphous film (see Ref. 18).

$\mathrm{Hg}_{1-x} \mathrm{Cd}_{x} \mathrm{Te}$ infrared detectors has been controlled in real time by SE to an accuracy better than $0.1 \% .^{9,10}$

The shape and position of electronic transitions also contain information regarding crystal structure. Sharp, welldefined absorption features rely on long-range order within a crystal. If this long-range order is disturbed, the absorption broadens and can shift in energy. This effect is used to estimate the degree of crystallinity in polysilicon films for display applications. ${ }^{11}$ The imaginary dielectric function for $\mathrm{SiC}$ is shown in Fig. 5. 4H-SiC has long-range hexagonal structure producing the well-defined absorption features. The hexagonal structure also exhibits a distinct uniaxial optical response for the electric fields oriented along the ordinary $(o)$ or extraordinary $(e)$ direction. ${ }^{12}$ The third curve shows an amorphous $\mathrm{SiC}$ film with broadened absorption shifted toward the red.

Both crystallinity and composition are derived from UV measurements, where large changes in optical properties occur at the electronic absorptions. Alternatively, IR wavelengths are important for conductivity measurements. Conductivity is related to available free carriers, which introduce Drude absorption at longer wavelengths. For conductive films, this absorption extends into the NIR. Figure 6 shows the free-carrier absorption for an indium-tin-oxide (ITO)

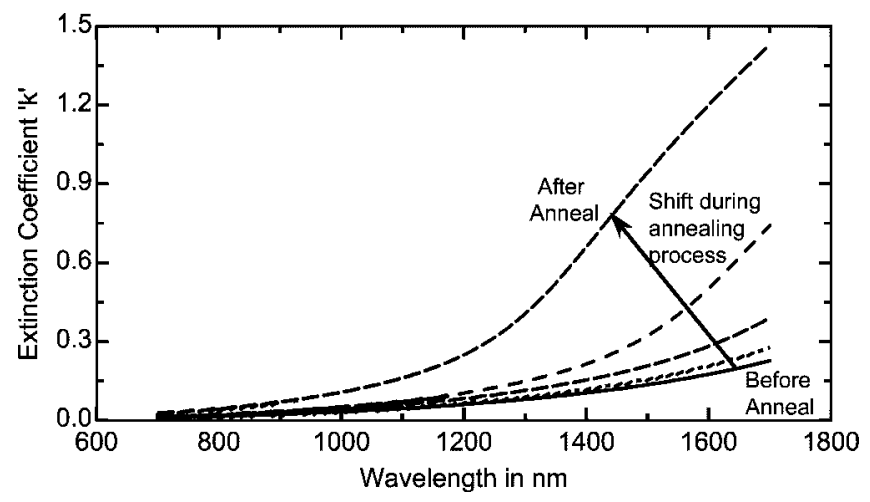

FIG. 6. SE measurements of ITO film during anneal process used to monitor conductivity by characterizing free-carrier absorption in the NIR. film used as a transparent conductor. It is desirable to increase conductivity (higher NIR absorption) while retaining visible transparency. The annealing process improved film quality as demonstrated by increasing NIR absorption. For less conductive films, longer wavelengths would be required, while more conductive films can be measured at shorter wavelengths. In either case, it can become difficult to separate conductivity from other absorption processes.

SE wavelengths are not always matched to a specific material property-they often coincide with applicationdependent wavelengths. For example, lasers are used by the photolithography, telecommunication, and data storage industries. Thus, thin films in these devices are best characterized at the application-specific wavelength. For photolithography, the exposure wavelength is in the UV: 248, 193, and now $157 \mathrm{~nm}$. It is important to measure optical constants at the exposure wavelength to properly understand interaction with photoresists, photomasks, and stepper-optic coatings. Telecommunication wavelengths are typically in the NIR. SE is used to characterize films and multilayer stacks used at each of the source and pump laser wavelengths (e.g., 850, 980, 1310, and $1550 \mathrm{~nm}$ ). Optical storage techniques use visible lasers to record information in phase-change media for DVD-R, CD-R, and CD-RW. SE characterization continues as lasers move toward the blue and UV to achieve higher storage densities. Thus, traditional SE from UV to NIR can characterize the films and stacks used with any of these device applications.

A single-wavelength ellipsometer may match the design wavelength, but fail to provide enough information. The additional information-content from SE over many wavelengths will help insure the correct answer at "design" wavelengths, along with other material properties of interest.

Further applications require a range of wavelengths to meet the application. Solar cells can be characterized at all wavelengths that respond to sunlight. Flat panel displays depend on visible optical properties to match their intended use. This is also true for eyeglass and window coatings.

Focus on wavelength range has led to exploration of regions not covered by traditional SE. Researchers in the 1980s demonstrated SE in both VUV ${ }^{13}$ and IR. ${ }^{14}$ However, these efforts were limited to a few research facilities until recent commercial SE development.

\section{VUV ELLIPSOMETRY}

VUV ellipsometry was first applied in Berlin at the BESSY synchrotron at photon energies up to $35 \mathrm{eV} .^{13}$ This system has an ultrahigh vacuum chamber to avoid the strong ambient absorption from oxygen and water vapor, while using light from the synchrotron. Recent characterization requirements for $157 \mathrm{~nm}$ lithography films led to the commercialization of VUV ellipsometry. These instruments feature nitrogen purge, $\mathrm{MgF}_{2}$ optics and a $\mathrm{D}_{2}$ source. ${ }^{15-17}$ This convenience has led to widespread use of VUV ellipsometry. Commercial instruments do not allow measurement above $9.5 \mathrm{eV}$. This is a new wavelength range for potential applications. 

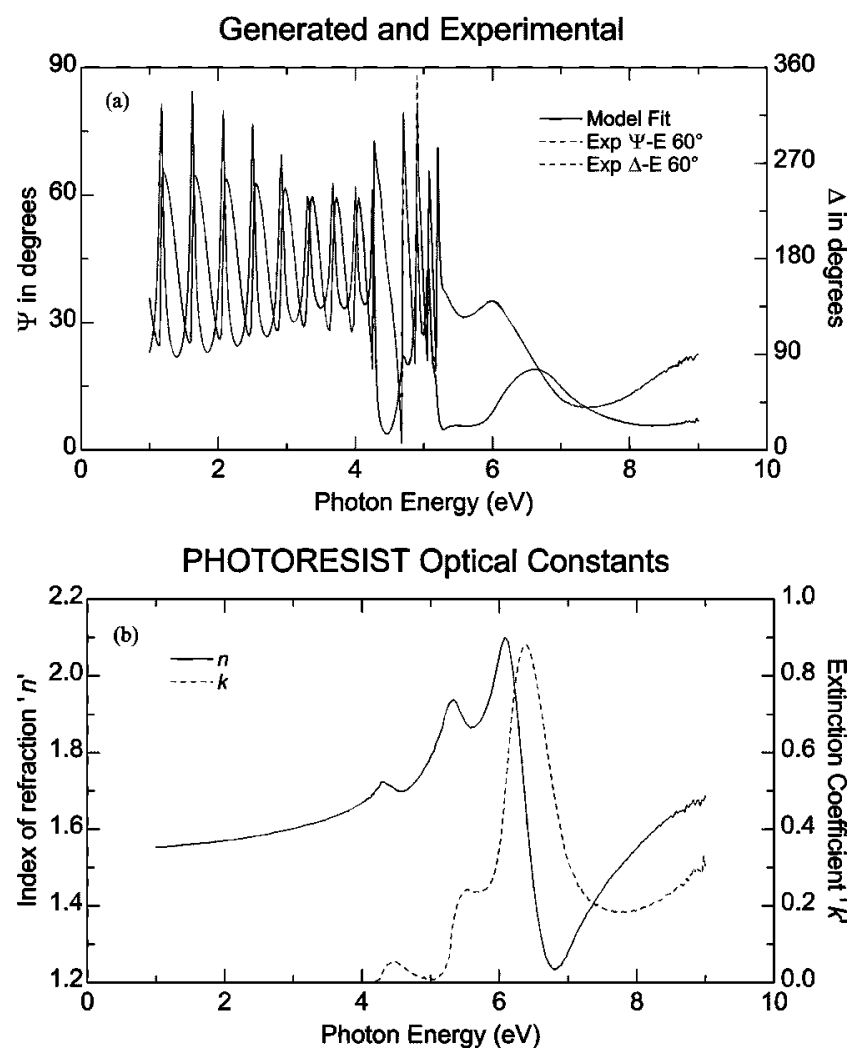

FIG. 7. (a) VUV ellipsometry measurement of photoresist film from $0.73 \mathrm{eV}$ $(1700 \mathrm{~nm})$ to $9.0 \mathrm{eV}(138 \mathrm{~nm})$. Resulting optical constants (b) were determined over this entire wavelength range.

A majority of VUV ellipsometry applications focus on lithography requirements. The lithography industry seeks to reduce feature sizes by moving the laser exposure to shorter wavelengths. This progression has led from 248 to $193 \mathrm{~nm}$ and now investigations of $157 \mathrm{~nm}$. The optical properties of all lithography films and bulk materials are critical to the overall performance. VUV ellipsometry has assisted the development of new materials for use at $157 \mathrm{~nm} .{ }^{18,19}$ The photoresists used at $193 \mathrm{~nm}$ are too absorbing for standard use at $157 \mathrm{~nm}$. Thus, new resist formulations are being developed with lower absorbance. In the meantime, $193 \mathrm{~nm}$ resists have been implemented in bilayer designs for early testing. ${ }^{20}$ Figure 7(a) shows the ellipsometry measurement of a photoresist deposited on silicon. This measurement was used to determine the photoresist optical constants shown in Fig. 7(b) up to $9 \mathrm{eV}(138 \mathrm{~nm})$.

The photoresist is often accompanied by an antireflective coating to suppress unwanted reflection from the substrate. A bottom antireflection coating (BARC) should provide the appropriate optical properties at $157 \mathrm{~nm}$ to match the photoresist. Typically, materials that can be "tuned" for different optical constants are incorporated as BARCs, including both organic and inorganic films. Once the optical properties are known for different process conditions, the lithography process can be optimized. ${ }^{21}$ Photomasks and pellicles are also important for lithography. VUV ellipsometry has been used to study new materials in these areas. ${ }^{22}$ Pellicles need to be very transparent, so thicker layers are used to gain high sen-

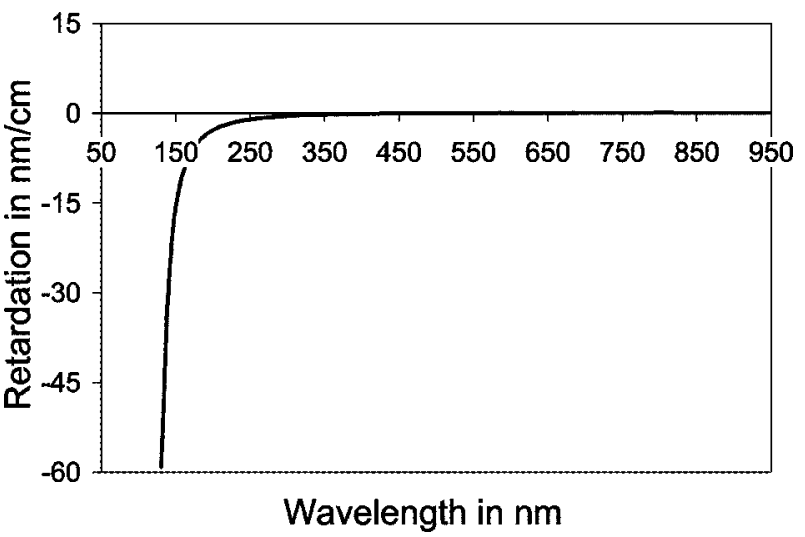

FIG. 8. Measured birefringence for $\mathrm{CaF}_{2}$ along the [110] direction (see Ref. 27).

sitivity to small absorption (longer path length increases sensitivity). Photomasks can incorporate an index gradient through the film. This allows the phase to be controlled and produce small linewidths. SE has been used to study index gradients in photomasks, but often requires more advanced measurement techniques. ${ }^{23,24}$ There are also many optical coatings applied to optics in exposure tools. These lens and mirror coatings are designed for antireflection or high reflection, so their optical properties and thickness are important. SE has been used to study different optical coatings, including many fluorinated films with low VUV absorption. ${ }^{25}$

Exposure tools use $\mathrm{CaF}_{2}$ optics for better transmission at $157 \mathrm{~nm}$. Low amounts of stress birefringence were found in original studies of cubic $\mathrm{CaF}_{2}$. However, these tests were performed at visible wavelengths. Intrinsic birefringence was discovered in cubic $\mathrm{CaF}_{2}$ along the [110] direction with strong wavelength dependence. ${ }^{26}$ This produces unacceptable birefringence levels for lithography optical elements. VUV ellipsometry confirmed this intrinsic birefringence. ${ }^{27,28}$ Figure 8 shows a measurement from $\mathrm{CaF}_{2}$ along the [110] direction. The birefringence measured at $157.6 \mathrm{~nm}$ is $-11.2 \mathrm{~nm} / \mathrm{cm}$. This is far above the maximum allowed in lithography lenses $(1 \mathrm{~nm} / \mathrm{cm})$, which was a major setback to $157 \mathrm{~nm}$ development.

VUV ellipsometry has also been used to characterize ultrathin films. There is an increased sensitivity to film thickness for shorter wavelengths. High- $\kappa$ gate dielectrics such as ultrathin $\mathrm{SiON}$ have been investigated with the desire to determine both thickness and nitrogen content. ${ }^{29}$ The most promise appears to be from a combination of characterization techniques to extract the most information.

The high photon energies of VUV ellipsometry also provide new information for the study of electronic transitions for wide-band gap materials. This study is of importance for the materials used in many optoelectronic, high-power, and high-temperature devices. SiC substrates have been well characterized by VUV ellipsometry. ${ }^{12,18,30}$ The electronic transitions in $\mathrm{SiC}$ occur at VUV photon energies. The anisotropy of these transitions is evident in Fig. 5 where ordinary and extraordinary dielectric functions are quite different. Many popular III-nitrides have also been measured with 


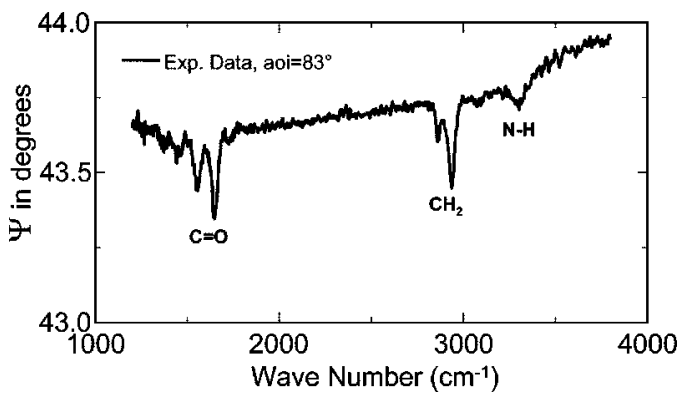

FIG. 9. IR-SE measurement of a thin Nylon film $(\sim 5 \mathrm{~nm})$ on $\mathrm{Au}$, with clearly identifiable molecular vibration absorptions (see Ref. 39 ).

VUV ellipsometry, including $\mathrm{GaN},{ }^{17} \mathrm{AlN},{ }^{31,32} \mathrm{AlGaN},{ }^{33,34}$ and AlInN. ${ }^{35}$

\section{IR ELLIPSOMETRY}

While IR ellipsometry has been around for decades, its applications have slowly developed. IR ellipsometry is plagued by nonideal sources, polarizers, compensators, and detectors. Thus, much of the progress for IR ellipsometry has come by way of improved optical designs, calibration, and correction procedures that allow accurate IR ellipsometry measurements. ${ }^{36}$ The IR region is sensitive to a variety of unique optical phenomena: free-carrier absorptions, molecular vibrations, and phonon absorptions.

Free-carrier absorption helps distinguish materials with different doping concentrations. At traditional SE wavelengths, epitaxial Si looks optically identical to an underlying Si substrate. In the infrared, this layer can easily be characterized provided the epitaxial layer has different doping concentration from the substrate. IR-SE at variable angles can provide information content for more complex characterization. It has been applied to doping implants ${ }^{37}$ and silicon

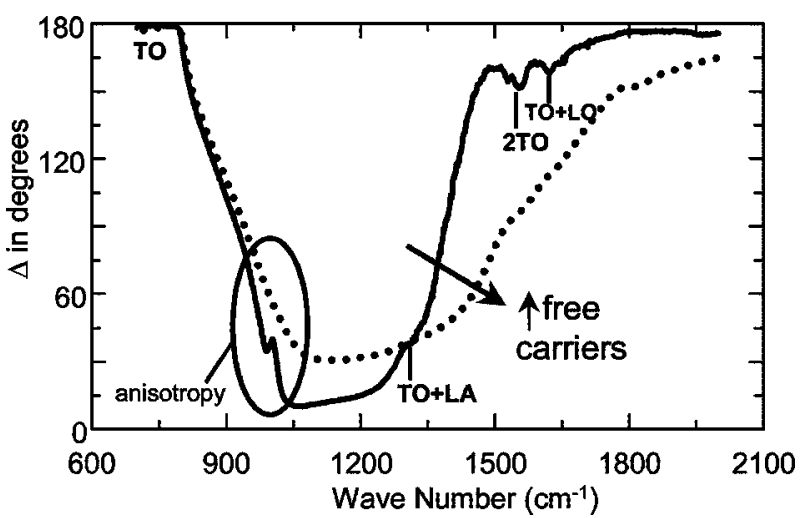

FIG. 10. IR-SE measurement of SiC, demonstrating the information content available in IR-phonon absorptions, free-carrier effects, and optical anisotropy.

membranes. ${ }^{38}$ In both cases, the doping concentration (and thus the optical properties) varied with depth through the material.

IR ellipsometry can also be applied to the study of molecular vibrations. Figure 9 demonstrates the sensitivity of IR ellipsometry to a $5 \mathrm{~nm}$ thick Nylon film on Au. ${ }^{39}$ The molecular vibrations are clearly evident in the spectra. In addition to molecular identification, it may also be possible to interpret the molecular orientation from IR-SE measurements. $^{40}$ IR-SE can also identify biological materials. $^{41,42}$ IR ellipsometry has demonstrated submonolayer sensitivity. ${ }^{43}$

Phonon absorptions often dominate the IR optical response of crystalline materials. Figure 10 shows the ellipsometric measurement from bulk SiC. The detailed spectra show two-phonon interactions and anisotropy in this hexagonal substrate. IR-SE has been applied to a wide range of materials. Recent attention has been directed toward many of the III-nitride compounds because of their application in
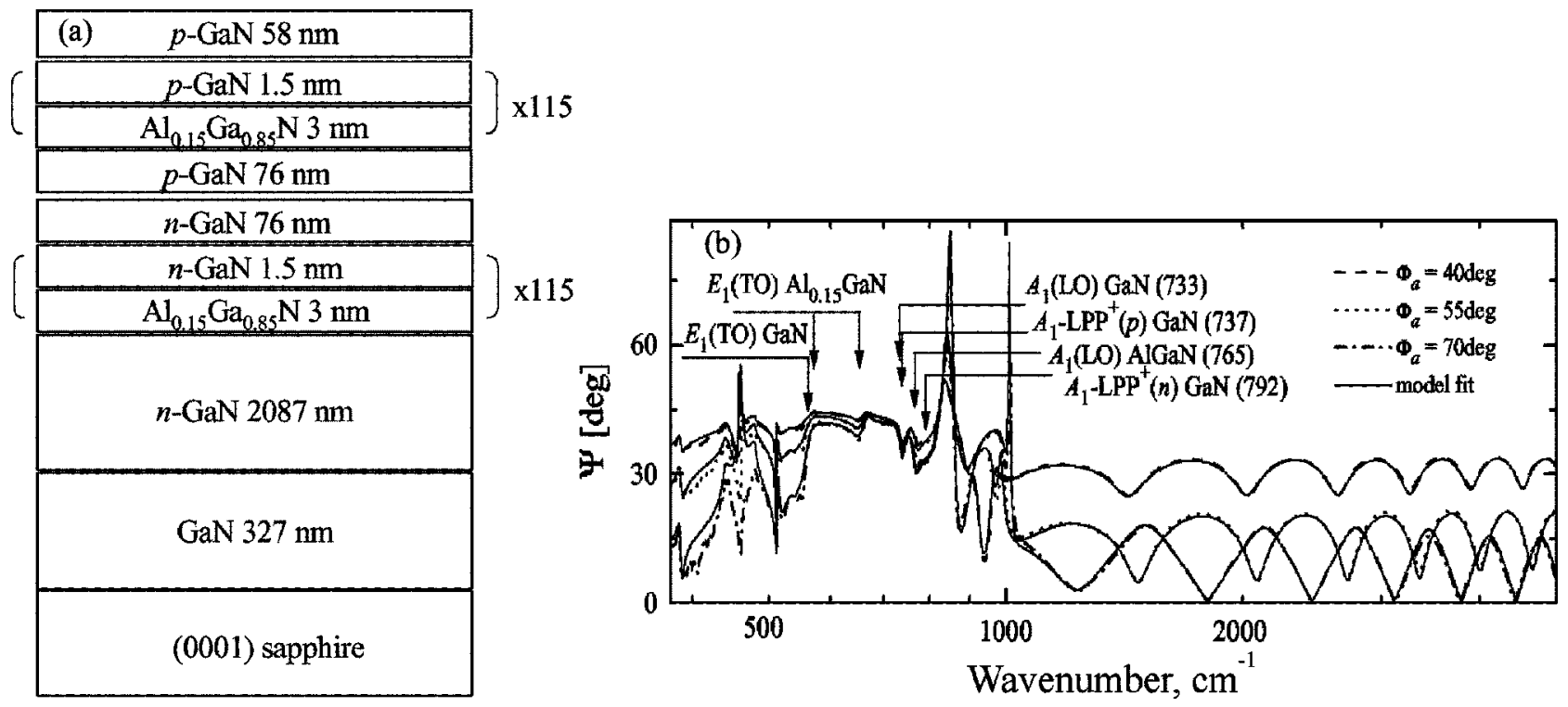

FIG. 11. (a) Model and (b) IR-SE measurement from a laser-diode structure. 
optoelectronic devices. The infrared optical response of these materials can be related to composition, lattice symmetry, lattice quality, and internal stress. In addition, anisotropic material properties can be investigated. IR-SE has been applied to $\mathrm{GaN},{ }^{44} \mathrm{AlGaN},{ }^{45} \mathrm{AlInN},{ }^{46}$ and superlattices of these materials. $^{47}$

Once the material properties are understood, this information can be applied to more complex structures used in optoelectronic devices. ${ }^{48,49}$ Figure 11(a) shows the details from a laser-diode model structure. The IR-SE measurement, shown in Fig. 11(b), was matched with this model to determine composition and strain information from the structure. $^{49}$

Other compound semiconductors have been investigated with IR-SE: InGaAsSb, ${ }^{50} \mathrm{GaAsN},{ }^{51} \mathrm{InGaAsN},{ }^{52} \mathrm{GaNP},{ }^{53}$ and AlGaInP. ${ }^{54}$ Superconductive $\mathrm{YBaCuO}^{55}$ and $\mathrm{YPrBaCuO}^{56}$ have also received recent attention with IRSE. Because the IR spectral region is rich with information, IR-SE will likely be applied to a growing number of new research areas.

\section{CONCLUSION}

Spectroscopic ellipsometry has been applied to a diverse range of applications pertaining to thin film and bulk material characterization. Traditionally, SE characterization was applied across the UV, visible, and NIR wavelength ranges. New developments have extended the available range to both the VUV and IR-opening the door to a range of applications. VUV ellipsometry is used to characterize $157 \mathrm{~nm}$ lithography materials, ultrathin films, and high photon energy electronic transitions. IR ellipsometry is sensitive to freecarrier, molecular-vibration, and phonon absorptions. Applications of SE that utilize wavelength-specific information were reviewed. In addition, the wavelength regions were discussed with a survey of application progress in these areas.

${ }^{1}$ R. M. A. Azzam and N. M. Bashara, Ellipsometry and Polarized Light, 2nd ed. (North Holland, Amsterdam, 1987).

${ }^{2} \mathrm{H}$. G. Tompkins, A User's Guide to Ellipsometry (Academic, San Diego, CA, 1993).

${ }^{3}$ H. G. Tompkins and W. A. McGahan, Spectroscopic Ellipsometry and Reflectometry (Wiley, New York, 1999).

${ }^{4}$ G. E. Jellison, Jr., Thin Solid Films 313-314, 33 (1998).

${ }^{5} \mathrm{~J}$. A. Woollam, in Wiley Encyclopedia of Electrical and Electronics Engineering, edited by J. G. Webster (Wiley, New York, 2000), p. 109.

${ }^{6}$ D. E. Aspnes, in Handbook of Optical Constants of Solids, edited by E. D. Palik (Academic, San Diego, CA, 1998), p. 89.

${ }^{7}$ T. E. Tiwald and M. Schubert, Proc. SPIE 4103, 19 (2000).

${ }^{8}$ S. Zollner et al., J. Appl. Phys. 88, 4102 (2000).

${ }^{9}$ B. Johs et al., Thin Solid Films 313-314, 490 (1998).

${ }^{10}$ J. Phillips, D. Edwall, D. Lee, and J. Arias, J. Vac. Sci. Technol. B 19, 1580 (2001)

${ }^{11}$ W. A. McGahan, B. R. Spady, B. D. Johs, and O. Laparra, Proc. SPIE 2725, 450 (1996).
${ }^{12}$ J. A. Woollam et al., Proc. SPIE 4099, 197 (2000).

${ }^{13}$ J. Barth, R. L. Johnson, and M. Cardona, in Handbook of Optical Constants of Solids II, edited by E. D. Palik (Academic, San Diego, CA, 1998), p. 213.

${ }^{14} \mathrm{~A}$. Roseler, Infrared Spectroscopic Ellipsometry (Akademie, Berlin, 1990).

${ }^{15}$ J. N. Hilfiker, B. Singh, R. A. Synowicki, and C. L. Bungay, Proc. SPIE 3998, 390 (2000).

${ }^{16}$ P. Boher et al., Proc. SPIE 3998, 379 (2000).

${ }^{17}$ S. Peters et al., J. Appl. Phys. 88, 4085 (2000).

${ }^{18}$ T. Wagner et al., Phys. Status Solidi A 188, 1553 (2001).

${ }^{19} \mathrm{~J}$. N. Hilfiker et al., Proceedings of the 1st International Symposium on $157 \mathrm{~nm}$ Lithography, 2000, p. 787.

${ }^{20}$ W. D. Kim et al., Proc. SPIE 4226, 93 (2000).

${ }^{21}$ J. N. Hilfiker, Semiconductor Fabtech 17, 87 (2002).

${ }^{22}$ R. H. French et al., Proc. SPIE 4346, 89 (2001).

${ }^{23}$ F. D. Kalk, R. H. French, H. U. Alpay, and G. Hughes, Proc. SPIE 2254, 64 (1994)

${ }^{24}$ B. Johs et al., Proc. SPIE 2253, 1098 (1994).

${ }^{25}$ B. Blanckenhagen, D. Tonova, and J. Ullman, Appl. Opt. 41, 3137 (2002).

${ }^{26}$ J. H. Burnett, Z. H. Levine, and E. L. Shirley, Phys. Rev. B 64, 241102/1 (2001).

${ }^{27}$ J. N. Hilfiker, Presented at the Proceedings of $157 \mathrm{~nm}$ Technical Data Review, Dallas, TX, 2002.

${ }^{28}$ J. N. Hilfiker and M. Suzuki, Optical Electro-Optical Engineering Contact 40, 225 (2002).

${ }^{29}$ P. Boher, P. Evrard, J. P. Piel, and J. L. Stehle, J. Non-Cryst. Solids 303, 167 (2002).

${ }^{30}$ O. P. A. Lindquist et al., Appl. Phys. Lett. 78, 2715 (2001).

${ }^{31}$ T. Wethkamp et al., Phys. Rev. B 59, 1845 (1999).

${ }^{32}$ M. P. Thompson, G. W. Auner, C. Huang, and J. N. Hilfiker, Mater. Res. Soc. Symp. Proc. 622, T6.5.1 (2001).

${ }^{33}$ A. Kasic et al., Phys. Rev. B 65, 184302/1 (2002).

${ }^{34}$ C. Cobet et al., Phys. Rev. B 64, 165203/1 (2001).

${ }^{35}$ A. Kasic et al., Mater. Res. Soc. Symp. Proc. 639, G6.13.1 (2001).

${ }^{36}$ U. S. Patent No. 5,706,212.

${ }^{37}$ T. E. Tiwald, D. W. Thompson, and J. A. Woollam, J. Vac. Sci. Technol. B 16, 312 (1998).

${ }^{38}$ E. Sossna et al., J. Vac. Sci. Technol. B 19, 2665 (2001).

${ }^{39}$ H. G. Tompkins, T. E. Tiwald, C. L. Bungay, and A. E. Hooper (unpublished).

${ }^{40}$ D. Tsankov et al., Langmuir 18, 6559 (2002).

${ }^{41}$ E. G. Caurel, B. Drévillon, and L. Schwartz, Proc. SPIE 4614, 134 (2002).

${ }^{42}$ E. Garcia-Caurel, B. Drévillon, A. De Martino, and L. Schwartz, Appl. Opt. 41, 7339 (2002)

${ }^{43}$ B. Drévillon, Thin Solid Films 313-314, 625 (1998).

${ }^{44}$ A. Kasic et al., Phys. Rev. B 62, 7365 (2000).

${ }^{45}$ A. Kasic, M. Schubert, J. Off, and F. Scholz, Appl. Phys. Lett. 78, 1526 (2001).

${ }^{46}$ M. Schubert et al., MRS Internet J. Nitride Semicond. Res. 4, 1 (1999).

${ }^{47}$ M. Schubert et al., Mater. Sci. Eng., B 82, 178 (2001).

${ }^{48}$ M. Schubert et al., Phys. Status Solidi B 228, 437 (2001).

${ }^{49}$ M. Schubert et al., Proc. SPIE 4449, 58 (2001).

${ }^{50}$ P. G. Snyder et al., Thin Solid Films 313-314, 667 (1998).

${ }^{51}$ G. Leibiger et al., J. Appl. Phys. 89, 4927 (2001).

${ }^{52}$ G. Leibiger, V. Gottschalch, and M. Schubert, J. Appl. Phys. 90, 5951 (2001).

${ }^{53}$ G. Leibiger, V. Gottschalch, A. Kasic, and M. Schubert, Appl. Phys. Lett. 79, 3407 (2001).

${ }^{54}$ T. Hofman et al., Phys. Rev. B 64, 155206/1 (2001).

${ }^{55}$ C. Bernhard et al., Solid State Commun. 121, 93 (2002).

${ }^{56}$ C. Bernhard et al., Phys. Rev. B 62, 9138 (2000). 